\title{
Ergebnisse, weiterführende Hypothesen und Fazit der Untersuchung
}

Die Studie „NGOs als besondere Akteure der Interessenvermittlung“ folgte der übergeordneten Zielsetzung, eine theoretisch-analytische Perspektive zu entwickeln, die ein differenzierendes Bild der Handlungs- und Entscheidungspraxis von NGOs als Organisationstypus zeichnete. Die Ergebnisse zeigen die Spannweite verschiedener Profilausprägungen im umfassenden Strategieportfolio moderner Interessenvermittlung in der deutschen NGO-Landschaft.

Als Ausgangspunkt und Richtschnur der Studie wurde ein konzeptionelles Dreieck der Handlungsrationalität im Kontext moderner Governance-Strukturen entwickelt. Die qualitativen Fallstudien zu fünf systematisch ausgewählten NGOs - Attac, LobbyControl, Foodwatch, Campact und die Deutsche Umweltstiftung (DUS) - decken dabei eine große Bandbreite von Erscheinungsformen in der deutschen NGO-Landschaft ab. Die Leitfrage, wie diese verschiedenen Typen von NGOs in öffentlichkeitswirksamen Prozessen der Interessenvermittlung agieren, folgte dem Forschungsziel der Hypothesengenerierung über die besondere politische Rationalität von NGOs. Anstatt theoretische Überlegungen zu testen oder verallgemeinerbare Aussagen über die Gesamtheit der NGOs zu treffen, zielte das Erkenntnisinteresse darauf, den breiteren Kontext von Handlungsstrategien der modernen Interessenvermittlung besser zu verstehen. Die Ergebnisbereiche umfassen zum einen die theoriegeleiteten Kategorien der Unterstützungs-, Einfluss- und Reputationslogik. Diese wurden zum anderen mit vier materialbasierten Kategoriensets ergänzt, die querliegende und mit der Strategieauswahl der Organisationen in Wechselwirkung stehende Aspekte enthalten: die übergeordnete Strategie, die Auswahl von zentralen Themen, die instrumentelle Verwendung von Expertise und die Legitimationsargumentation. 
NGO-spezifische Besonderheiten der Interessenvermittlung

$\mathrm{Zu}$ den NGO-spezifischen Besonderheiten zählen (1) der ausgeprägte Bezug zu Fachexpertise als politisches und legitimatorisches Instrument im eigenen Selbstverständnis, (2) flexible Organisationsstrukturen, um sich den Anforderungen moderner Politikvermittlung in Governance-Strukturen anzupassen, (3) verschiedene Ansätze der Unterstützer*innen-Mobilisierung, um dem punktuellen und zeitlich begrenzten Engagement vor dem Hintergrund nichtmitgliedschaftsbasierten Finanzierungsformen gerecht zu werden sowie (4) besondere Ansprüche an den Zuschnitt von Themen und Forderungen im Spannungsfeld von Glaubwürdigkeit, politischer Anerkennung als relevanter Akteur und dem (begrenzten) Erfolg in Politikwandelprozessen. Zusammengenommen zeigen sich (5) Abwägungsprozesse von vielschichtigen, teils widersprüchlichen Anforderungen von Basis, Medien und Politik, die sich in ähnlicher Form in den Veränderungstrends der politischen Interessenvermittlung von Verbänden wiederfinden.

Binnendifferenzierung von „NGOs“ als Subtypen von public interest Organisationen

An diesen Besonderheiten zeigt sich, welche verschiedenen Strategietypen und Organisationszuschnitte unter das Label „NGO“ fallen. Dieses hohe Maß an Heterogenität, welches der vermeintliche Sammelbegriff subsumiert, lässt daran zweifeln, ob sich das weitgefasste NGO-Verständnis plausibel verwenden lässt. Vielmehr legen die Ergebnisse der vorliegenden Analyse den Schluss nahe, dass es nicht sinnvoll ist, von , $N G O s^{\prime}$ ' in der politischen Interessenvermittlung zu sprechen. Alternativ eignet sich eine Binnendifferenzierung von unterschiedlichen Subtypen von public interest Organisationen (Abbildung 7.1).

Aus den Ergebnissen lassen sich vier ausdifferenzierte, eigenständige NGOSubtypen herauslesen. Ein erster Subtyp orientiert sich als (1) Mitgliedschafts$N G O$ stark an basisdemokratischen Handlungsmustern. Die Organisation setzt auf große Mitgliederzahlen, aktive Beteiligung und basisdemokratische Mitentscheidung bei politischen Aktivitäten. Sie fühlt sich - nicht anders als klassische Mitgliedsverbände - in besonderer Weise den Mitgliedern und Unterstützer*innen im Sinne einer erweiterten Mitgliedschaftslogik verpflichtet. Im zweiten Subtyp der (2) Expertise-NGO bilden Wissen und Fachexpertise die Basis der Organisation. Beide können als Währung in der Kommunikation mit Politik und Öffentlichkeit eingesetzt werden, mit der sich die Organisation im politischen Prozess profiliert. Der dritte Subtyp der (3) Kampagnen-NGO setzt in der Strategiewahl und der Organisationsausrichtung die Akzente auf Kampagnen- und 


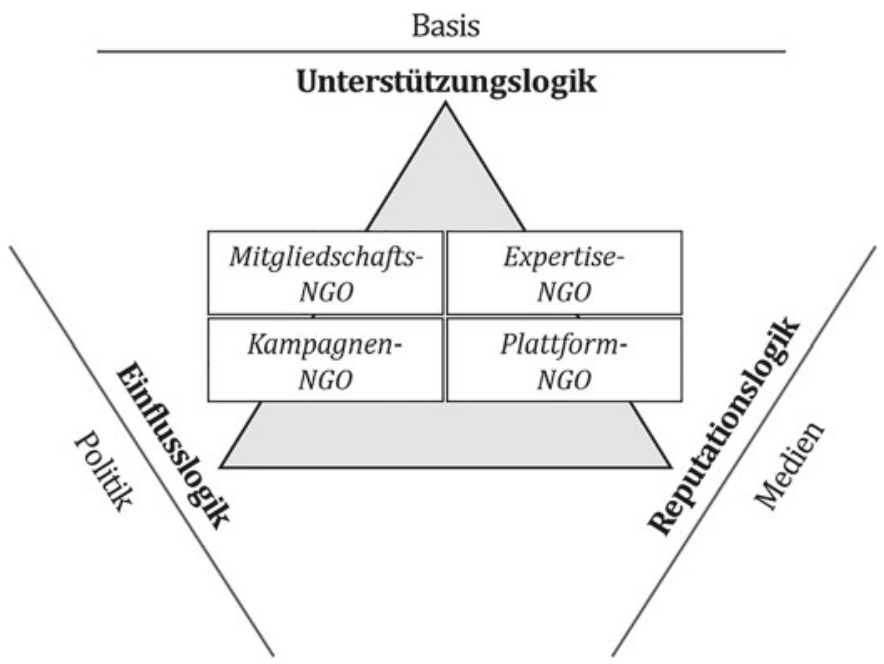

(Grafik: eigene Darstellung)

Abbildung 7.1 Profilausprägungen von NGO-Strategieportfolios

Mobilisierungsarbeit. Öffentliche Aufmerksamkeit und strategisches Themensetzen - welches über Watchdog-Funktionen bis hin zur Skandalisierung reicht - sind hier der Goldstandard. Denn Sichtbarkeit ist die Grundlage, auf der die Legitimation dieser Organisation fußt. Im Kontext der professionalisierten Interessenvermittlung ähneln die Subtypen Zwei und Drei den etablierten Verbänden, darunter insbesondere public interest Verbänden, im praktischen Umgang mit Medien und politischen Entscheidungsträger*innen. Der vierte Subtyp einer (4) Plattform-NGO lässt sich als professionalisierte Protest- und Kampagnenplattform charakterisieren, der einem fluiden Kreis von Interessierten Mobilisierungsangebote zu punktuellen Themen macht. Vereinfacht gesprochen bietet die Organisation eine Bühne, das Stück spielen andere. Sie knüpft und pflegt die Vernetzungen, welche die Zivilgesellschaft als solche aktiv in Erscheinung treten lässt. Da dieser Typ in der idealisierten Form lediglich eine Art Rumpfverein ausbildet, weicht er am stärksten von den Standards der public interest Interessenorganisationen ab.

Die Analyse aus der Perspektive des Dreiecks der Handlungsrationalität verdeutlicht, dass die Organisationen aus einem vielschichtigen Strategieportfolio 
wählen können. Dies deckt sich mit einem wachsenden Kanon von Forschungsergebnissen, die postulieren, dass die Strategiewahl nicht durch den Organisationstyp determiniert wird, sondern sich nach einem weiteren Kriterienkatalog richtet. Dieser schlägt sich u. a. in den drei Handlungslogiken und den vier identifizierten Sets der Strategie, Themenwahl, Expertise und Legitimation nieder. Die Untersuchungsergebnisse schließen an die weiterführende Frage an, ob diese Organisationsstrategien ein Zeichen einer NGO-isierung der Interessengruppenlandschaft ist (vgl. Lang 2013, Choudry/Kapoor 2013, Paternotte 2016) oder ob die Interessengruppen damit auf Herausforderungen und Veränderungstrends der politischen Interessenvermittlung in Governance-Strukturen reagieren (vgl. Lösche 2007, Strünck/Sack 2017).

\section{Skizze einer zukünftigen Forschungsagenda}

Das Forschungsprojekt „NGOs und Politikmanagement“, welches dieser Publikation zugrunde liegt, wurden durch die Drittmittelfinanzierung des Gemeinschaftsausschusses der Deutschen Gewerblichen Wirtschaft möglich gemacht. Dies diente als Anschubfinanzierung, den Forschungsbereich an der NRW School of Governance weiterzuführen und zukünftig ausbauen zu können.

In der empirischen Praxis hat sich die Kombination aus Forschungspuzzle, Leitfrage, theoretischer Konzeption und den skizzierten Ergebniskategorien bewährt. Der differenzierende Blick aus der Perspektive des Dreiecks der Handlungsrationalität erwies sich als flexibel und anschlussfähig, um die Besonderheiten und Strategiemöglichkeiten der systematisch ausgewählten NGOs aufzuzeigen. Diese Elemente können als wissensbasiertes Gerüst dienen, um weiterführende Fragestellungen in die Breite und die Tiefe des Themenfelds zu formulieren.

Eine erste Option für weiterführende qualitative Forschung liegt darin, die Fallauswahl und das Datenmaterial zu erweitern. So können etwa die in der Studie identifizierten TOP-10 Organisationen sowie weitere große Verbände und Akteure einbezogen werden, die sich am Kristallisationspunkt der Auswahlstrategie, der Gemeinschaftskampagne gegen TTIP und CETA, beteiligten. Zudem können als weitere Kristallisationspunkte die Mobilisierungsmomente der Gemeinschaftskampagnen im Umfeld von Fridays for Future ergänzt werden. Nach dem erfolgreichen Praxistest der analytischen Kategorien können zudem NGOs und public interest Organisationen in weiteren pluralistisch geprägten Politikfeldern untersucht werden. Für die Auswahl sind insbesondere Organisationen mit Maximalausprägungen in der Spannweite der Kategoriensets relevant. Ein zentraler Schritt ist die Erweiterung des Datenmaterials von derzeit über 30 
Experteninterviews und über 15 Podcast-Interviews. Neben weiteren Experteninterviews können zusätzliche Podcast-Hintergrundgespräche genutzt werden, wie auch politische Stellungnahmen und Pressemitteilungen.

Eine zweite Option erweitert den Methodenpool der qualitativen Textanalyse um quantitative Verfahren, wie data mining und quantitative text analysis (u. a. Blätte/Behnke et al. 2018, Sack 2017, Aizenberg 2020). Diese Methoden richten den Blick auf die Gesamtpopulation eines Politikfeldes und die übergeordnete Ebene des diskursiven Austausches und der Kontaktbeziehungen zwischen NGOs und Medien bzw. zwischen NGOs und politischen Entscheidungsträger*innen. Durch eine netzwerkanalytische Brille ist es möglich, die personelle oder organisationale Verzahnung der NGO- bzw. Interessengruppenlandschaft nachzuzeichnen. Interessant wäre hier, ob sich die Häufigkeit von Themenkooperation und Projektfinanzierungen auch im Personalwechsel der professionalisierten Mitarbeiter*innen niederschlägt. Mit einem ausreichend hohen $\mathrm{Maß}$ an qualitativem und quantitativem Datenmaterial und deutlich definierbaren Policy-Outcomes lässt sich außerdem die Methode der Qualitative Comparative Analysis (QCA) einsetzen, um zu untersuchen, inwieweit sich die verschiedenen Austauschstrukturen der Politikfelder in theoretisch-konzeptioneller Hinsicht (begrenzt) generalisieren lassen.

Neben der Fortführung dieses Forschungsansatzes kann die zukünftige Forschungsagenda neue Themenhorizonte und verschiedene Analyseebenen erschließen. Diese Ebenen umfassen sowohl die Binnenorganisation und Entscheidungsprozesse innerhalb von NGOs, die Beziehungen zwischen NGOs und Bürger*innen, als auch die Kontakt- und Austauschbeziehungen zu politischen Entscheidungsträger*innen in Regierung, Parlament und Verwaltung. Auf Ebene der Intermediären werden zudem die Interaktionen zwischen NGOs und anderen Akteuren der Interessenvermittlung, wie Verbänden, Unternehmen, Gewerkschaften, Stiftungen, Think Tanks einbezogen. (1) Ein Themenschwerpunkt auf der internen Dimension der Organisationspraxis kann die Frage stellen, wie und in welchem Umfang sich Unterschiede in Strategiewahl und Strategiefähigkeit durch das Organisationsformat erklären lassen. Relevant ist in diesem Schwerpunkt, welche internen Integrations- und Koordinationsleistungen die NGOs im Zusammenhang mit der Wahl von Bündnispartnern und Arenen der Interessenvermittlung erbringen. (2) Ein Schwerpunkt auf der externen Dimension kann thematisieren, in welchem Ausmaß staatliche Akteure als Regulatoren und Prozessgestalter einen gesetzgeberischen oder informellen Einfluss auf die Aktivitäten von NGOs und anderen Interessengruppen nehmen. Insbesondere lassen sich die Veränderungstrends der Interessenlandschaft in Bezug auf die Auswirkungen der Digitalisierung auf die Prozesse der Mobilisierung, Netzwerkbildung 
und Interessenaggregation nachzeichnen. (3) Hinsichtlich eines demokratietheoretischen und normativen Schwerpunkts lassen sich Optionen herausarbeiten, um kritische Entwicklungen im Feld der Zivilgesellschaft bezüglich rechtsradikaler und menschenfeindlicher Bewegungen zu adressieren (siehe Abschnitt 2.2), die sich nicht am Gemeinwohl orientieren. Bei diesen drei Schwerpunkten des Themenhorizonts stellt ausgerechnet die definitorische Unschärfe des Sammelbegriffs „NGOs“ eine forschungspragmatische Chance für eine verbesserte Anschlussfähigkeit der Forschung dar. Einerseits ergänzt die Binnendifferenzierung verschiedener Subtypen von public interest Organisationen die Gestaltung bisheriger Studiendesigns. Andererseits rücken NGOs auch politisch und medial vermehrt ins Rampenlicht. Aktuelle Aufmerksamkeitswellen folgen den genannten zivilgesellschaftlichen Mobilisierungserfolgen, der politischen Debatte um die gesetzliche Neuregelung von Gemeinnützigkeit im Nachgang an das Attac-Urteil des Bundesfinanzhofs Anfang 2019 sowie der Frage, wie sich die Bewegungen wie Fridays for Future oder Pegida auf konkrete Politikergebnisse auswirken. Hier ist zu erwarten, dass neue Forschungskonjunkturen ebenso die Erkenntnisse zu NGOs in der Interessenvermittlung steigern.

Zusammengenommen zeigen die Untersuchungsergebnisse, wie facettenreich die Handlungspraxis von NGOs im weiteren politischen Umfeld ist. Eine systematisch ausgerichtete, politikwissenschaftliche Perspektive kann die verschiedenen Wissensstände in einen gemeinsamen Kontext setzen und neue Erkenntnisse für die Regierungs- und Interessengruppenforschung liefern. Sie trägt dazu bei, die Gestaltungsperspektive des Politikmanagements in den Besonderheiten des Strategieportfolios moderner Interessenvermittlung zu erschließen.

Open Access Dieses Kapitel wird unter der Creative Commons Namensnennung 4.0 International Lizenz (http://creativecommons.org/licenses/by/4.0/deed.de) veröffentlicht, welche die Nutzung, Vervielfältigung, Bearbeitung, Verbreitung und Wiedergabe in jeglichem Medium und Format erlaubt, sofern Sie den/die ursprünglichen Autor(en) und die Quelle ordnungsgemäß nennen, einen Link zur Creative Commons Lizenz beifügen und angeben, ob Änderungen vorgenommen wurden.

Die in diesem Kapitel enthaltenen Bilder und sonstiges Drittmaterial unterliegen ebenfalls der genannten Creative Commons Lizenz, sofern sich aus der Abbildungslegende nichts anderes ergibt. Sofern das betreffende Material nicht unter der genannten Creative Commons Lizenz steht und die betreffende Handlung nicht nach gesetzlichen Vorschriften erlaubt ist, ist für die oben aufgeführten Weiterverwendungen des Materials die Einwilligung des jeweiligen Rechteinhabers einzuholen.

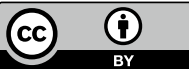

